\title{
In vitro microdialysis membrane efficiency of broad-spectrum antibiotics in combination and alone
}

This article was published in the following Dove Press journal:

Clinical Pharmacology: Advances and Applications

5 June 2014

Number of times this article has been viewed

\author{
Shawn H MacVane' \\ Seth T Housman' \\ David P Nicolau',2 \\ 'Center for Anti-Infective Research \\ and Development, ${ }^{2}$ Division of \\ Infectious Diseases, Hartford Hospital, \\ Hartford, CT, USA
}

Purpose: Microdialysis is a valuable technique for studying the distribution of drugs into interstitial fluid, the target site for a pharmacologic effect. Due to incomplete equilibrium, retrodialysis is a method used to correct for relative recovery. The impact of two-drug combinations on probe recovery, however, remains unknown.

Methods: In vitro microdialysis was conducted for five antibiotics (avibactam, cefepime, ceftaroline, piperacillin-tazobactam, and vancomycin), representing three empiric antimicrobial regimens, to assess the impact of two-drug combinations on probe recovery. Recoveries were compared between single and two-drug treatments.

Results: Recoveries by gain and loss were linear with their molecular weight. During all gain experiments, recoveries were similar when tested alone or in combination with another antibiotic. Unacceptable differences in recovery by loss were observed for cefepime in the presence of vancomycin $(-21 \%)$ and vancomycin in the presence of piperacillin-tazobactam $(-22 \%)$.

Conclusion: Differences among in vitro recovery by loss suggest two-drug combinations may impact dialysate recovery during in vivo retrodialysis procedures, particularly when larger molecular weight drugs (ie, vancomycin) are involved. Importantly, there were no differences during gain experiments. In vitro studies, as performed here, should be conducted for each potential two-drug combination, prior to their combined use for in vivo retrodialysis.

Keywords: microdialysis, retrodialysis, combination therapy

\section{Introduction}

In vivo microdialysis has become a valuable sampling technique for the study of drug distribution into interstitial fluid. Alternatively, microdialysis can be used as a delivery method by adding the drug to the perfusion fluid. ${ }^{1}$ The microdialysis methodology has been previously described in detail. ${ }^{2-5}$ Briefly, a probe containing a semi-permeable dialysis membrane is implanted into the tissue of interest and continuously perfused with a physiological solution (perfusate). Diffusible (unbound) substances are exchanged into the probe, assuming a concentration gradient exists, resulting in their presence in the solution perfusing the probe (dialysate).

During microdialysis sampling studies, the probe is continuously perfused, making the dialysate concentration only a fractional recovery of the actual tissue concentration, termed the relative recovery. As a quantifiable concentration in tissue is desired, the probe must be calibrated in vivo to account for the fractional recovery. ${ }^{4}$ The retrodialysis by drug method, as used during recovery by loss experiments, is the most commonly used calibration method in human microdialysis studies. ${ }^{4}$ This method uses a calculation,
Center for Anti-Infective Research and Development, Hartford Hospital, 80 Seymour Street, Hartford,

CT 06102, USA

Tel + I 860545394 I

$\mathrm{Fax}+\mathrm{I} 8605453992$

Email david.nicolau@hhchealth.org 
derived from the loss of drug from the perfusate during in vivo retrodialysis, to correct for the in vivo recovery.

While advantageous for many reasons, this method fails to address many practical issues, such as the relationship between multiple antimicrobial agents that may be required for appropriate empiric coverage in many infection types, such as skin and skin structure infections. A number of elements are known to influence the relative recovery, of which the characteristics of solute and the tissue prohibit the use of in vitro calibration as a surrogate for calibration in vivo. ${ }^{5}$ Although in vitro experiments will not eliminate the need for in vivo recoveries, these experiments provide essential information on the solute gain and loss and the feasibility of calibration in vivo. Supratherapeutic concentrations, often several times higher than those observed in patients, are required during retrodialysis. The presence of two drugs in the perfusate during the retrodialysis procedure may alter the composition characteristics of the fluid. Therefore, when microdialysis is used for pharmacokinetic studies of new pharmacological agents, in vitro gain (relative recovery) and loss (retrodialysis) experiments are often performed prior to application in humans. These studies provide preliminary information on solute recovery and feasibility of in vivo calibration. ${ }^{4}$ Given the magnitude of effect the retrodialysis procedure ultimately has on determining the in vivo tissue concentrations, we sought to evaluate if probe recovery of empiric antimicrobial agents is affected by use of two drugs in combination during in vitro microdialysis experiments.

\section{Materials and methods Study design}

In an attempt to reduce the uncertainty of whether the presence of multiple antibiotics affects the recovery of one another during retrodialysis, we performed in vitro mono- and combination therapy experiments evaluating the probe recovery by gain and loss over a 3 hour period for the following agents: avibactam (AVI), cefepime (FEP), ceftaroline fosamil (CPT), piperacillin-tazobactam (TZP), and vancomycin (VAN).

\section{Antimicrobial test agents}

Commercially available cefepime, ceftaroline fosamil, piperacillin-tazobactam, vancomycin, and analytical-grade avibactam (lot AFCH005151; Forest Laboratories, Inc., New York, NY, USA) were utilized for all studies.

Analyte of interest $\left(A_{i}\right)$ solutions were freshly prepared the morning of loss experiments and were frozen overnight for gain experiments. To account for potential concentration changes from the freeze-thaw process, $\mathrm{A}_{\mathrm{i}}$ solutions were assayed hourly during gain experiments. $A_{i}$ concentrations were chosen to simulate in vivo retrodialysis, approximately 10-20 times the trough serum concentrations in clinically treated patients (Table 1).

\section{Recovery procedures}

Three microdialysis probes (CMA 60 microdialysis catheters; CMA Microdialysis AB, Solna, Sweden), with membrane lengths of $30 \mathrm{~mm}$ and a molecular mass cutoff of 20 $\mathrm{kDa}$, were connected to a microinfusion pump (CMA 107 microdialysis pump). Once submerged, the catheters were flushed at $15 \mu \mathrm{L} / \mathrm{min}$ for 5 minutes, then continuously perfused with $\mathrm{A}_{\mathrm{i}}$ (loss) or lactated Ringer's solution (LR) (gain) at $2 \mu \mathrm{L} / \mathrm{min}$. Dialysate samples of $120 \mu \mathrm{L}$ were obtained hourly from the three catheters. There was a 1 hour washout between treatments to allow multiple probe flush sequences with each new solution, to ensure no carryover of the previous solution remained in the tubing. Dialysate samples were collected in $200 \mu \mathrm{L}$ microvials (CMA Microdialysis

Table I Tested drugs and mean recovery by gain and loss when administered alone and in combination with another antibiotic

\begin{tabular}{llllllll}
\hline Drug tested & $\begin{array}{l}\text { Molecular } \\
\text { weight }(\mathbf{D a})\end{array}$ & $\begin{array}{l}\text { Targeted } \\
\text { concentration } \\
(\mathbf{m g} / \mathbf{L})\end{array}$ & $\begin{array}{l}\text { Combination } \\
\text { drug }\end{array}$ & $\begin{array}{l}\text { Gain alone } \\
\text { (mean } \pm \text { SD, } \\
\% \text { recovery) }\end{array}$ & $\begin{array}{l}\text { Gain combination } \\
\text { (mean } \pm \text { recovery) }\end{array}$ & $\begin{array}{l}\text { Loss alone } \\
\text { (mean } \pm \text { SD, } \\
\% \text { recovery) }\end{array}$ & $\begin{array}{l}\text { Loss combination } \\
\text { (mean } \pm \text { recovery) }\end{array}$ \\
\hline AVI & 287.23 & 10 & CPT & $76.1 \pm 11.0$ & $85.3 \pm 12.3$ & $80.9 \pm 3.0$ & $83.9 \pm 2.3$ \\
CPT & 762.8 & 20 & AVI & $69.8 \pm 7.8$ & $68.4 \pm 6.0$ & $63.4 \pm 3.8$ & $60.7 \pm 2.8$ \\
FEP & 571.50 & 200 & VAN & $58.0 \pm 6.7$ & $59.5 \pm 5.1$ & $73.5 \pm 4.3$ & $52.5 \pm 8.3$ \\
VAN & $1,485.74$ & 200 & FEP & $38.7 \pm 5.1$ & $50.5 \pm 5.6$ & $45.7 \pm 7.4$ & $49.4 \pm 3.7$ \\
PIP (in presence & 539.5 & 400 & VAN & $51.6 \pm 7.1$ & $46.0 \pm 8.6$ & $62.0 \pm 5.4$ & $74.0 \pm 5.2$ \\
of TAZ) & & & & & & $75.7 \pm 7.5$ & $83.2 \pm 4.5$ \\
TAZ (in & 322.3 & 50 & VAN & $61.0 \pm 11.8$ & $54.8 \pm 14.4$ & \\
Presence of PIP) & & 200 & TZP & $46.0 \pm 4.7$ & $55.9 \pm 8.5$ & $56.3 \pm 3.1$ & $33.8 \pm 7.7$ \\
VAN & $1,485.74$ & 200 &
\end{tabular}

Notes: ${ }^{\dagger}$ Target concentration represents the starting concentration of $C_{\text {medium }}$ (recovery by gain) and $C_{\text {perfusate }}$ (recovery by loss).

Abbreviations: AVI, avibactam; CPT, ceftaroline fosamil; FEP, cefepime; PIP, piperacillin; TAZ, tazobactam; TZP, piperacillin-tazobactam; VAN, vancomycin; SD, standard deviation. 
$\mathrm{AB}$ ), which were stored in amber polypropylene tubes and immediately frozen at $-80^{\circ} \mathrm{C}$ until analysis.

For recovery by gain, probes $(n=3)$ were placed into $10 \mathrm{~mL}$ LR solution containing $\mathrm{A}_{\mathrm{i}}$ at concentration $\left(\mathrm{C}_{\text {medium }}\right)$ and perfused with blank LR. The level of gain for individual probes was calculated based on the concentration of the dialysate sample collected $\left(\mathrm{C}_{\text {dialysate }}\right)$ compared with the concentration of the stock solution using the following: percent recovery by gain $=\left(\mathrm{C}_{\text {dialysate }} / \mathrm{C}_{\text {medium }}\right) \times 100$.

For recovery by loss, probes $(\mathrm{n}=3)$ were placed into $10 \mathrm{~mL}$ blank LR solution and perfused with a solution of $\mathrm{A}_{\mathrm{i}}$ at concentration $\left(\mathrm{C}_{\text {perfusate }}\right)$, and its rate of disappearance through the membrane was determined as the level of recovery. The loss for individual probes was calculated using the following equation: percent recovery by loss $=\left[\left(\mathrm{C}_{\text {perfusate }}-\mathrm{C}_{\text {dialysate }}\right) / \mathrm{C}_{\text {perfusate }}\right] \times 100$.

Each antibiotic was tested for 3 hours followed by a solution of the two drugs in combination. For example: ceftaroline fosamil alone, avibactam alone, then a combination of ceftaroline fosamil-avibactam. Experiments were conducted in triplicate (three separate catheters) over 3 hours (ie, nine repeated observations) to assess possible changes in recovery over time and to minimize the chance of random sampling error.

\section{Analytical procedures}

Concentrations in solution and dialysate (cefepime, piperacillintazobactam, and vancomycin) were determined using a validated high-performance liquid chromatography (HPLC) assay that was developed using a previously published assay at the Center for Anti-Infective Research and Development, ${ }^{6-8}$ while ceftaroline fosamil and avibactam concentrations were determined by Eurofins Medinet, Inc. (Chantilly, VA, USA) using a previously described liquid chromatography-tandem mass spectrometry assay. ${ }^{9}$ The mean interday and intraday coefficients of variation for high and low check samples were $\leq 10 \%$ for individual assays. Data are represented as the mean $\pm \mathrm{SD}$ recoveries of the nine independent observations for each treatment.

Mean recoveries for each $A_{i}$ were compared between studies to calculate the treatment difference, defined as the change in percent recovery (by gain or loss) between mono- and combination therapy treatments. A change of $>20 \%$ in mean recovery was deemed an unacceptable level of difference between treatments. This value was determined to represent a change in recovery greater than would be expected from the variability of the system.

\section{Results}

Mean recovery by gain and loss of tested drugs ranged from $34 \%-85 \%$ (Table 1) and was linear with their molecular weight ( $R^{2}=0.60$ and $R^{2}=0.81$, respectively). There were no changes in recovery over the 3 hour sample period and between the three catheters for each of the tested drugs (data not shown). Recoveries were similar ( $\leq 20 \%$ difference) when tested alone or in combination therapy regimens, (Figure 1) for avibactam ( \pm ceftaroline fosamil), ceftaroline fosamil ( \pm avibactam), and piperacillin-tazobactam ( \pm vancomycin) during gain and loss studies. Unacceptable differences during loss experiments were observed for cefepime \pm vancomycin $(-21 \%)$ and vancomycin \pm piperacillin-tazobactam $(-22.5 \%)$ (Figure 1B).

\section{Discussion}

Microdialysis is a useful approach for obtaining pharmacokinetic data for decision support in antimicrobial dosing design. ${ }^{10}$ While in vitro microdialysis has previously been used to evaluate microdialysis probe recovery, to the best of our knowledge, this is the first experiment to determine the efficiency of recovery among broad-spectrum antibiotics alone and in combination with another agent. Given the increased prevalence of multidrug-resistant pathogens and the need to cover for Gram-positive and -negative organisms, combination therapy is often employed to improve the adequacy of empiric therapy. ${ }^{11,12}$ Additionally, development efforts of new antibacterial agents have focused largely on the use of $\beta$-lactamase inhibitors to battle resistance. ${ }^{13,14}$ Therefore, knowledge of potential interactions among compounds during the retrodialysis procedure, if any, is of important value for future microdialysis studies. With retrodialysis being the measurement that ultimately determines the degree of correction for probe recovery, it is arguably, the most influential piece of data collected during microdialysis studies. If this recovery varies substantially between single and two-drug combinations, the calculated concentration for the studied antibiotic will over- or underestimate the actual tissue exposure, depending on the magnitude (positive or negative change) of the difference. This circumstance, in turn, will lead to inaccurate determination of antibiotic exposure in the interstitial space fluid. Furthermore, as retrodialysis has become a popular method of local drug delivery to the skin, potential interactions between administered agents could result in unintended toxicity or therapeutic failure.

During in vitro recovery studies of five antibiotics, we observed a wide range of differences between single and two-drug combinations from $-22 \%$ to $12 \%$. The greatest differences were seen in loss experiments containing vancomycin, including the two unacceptable findings of cefepime \pm vancomycin and vancomycin \pm piperacillin-tazobactam. Given 


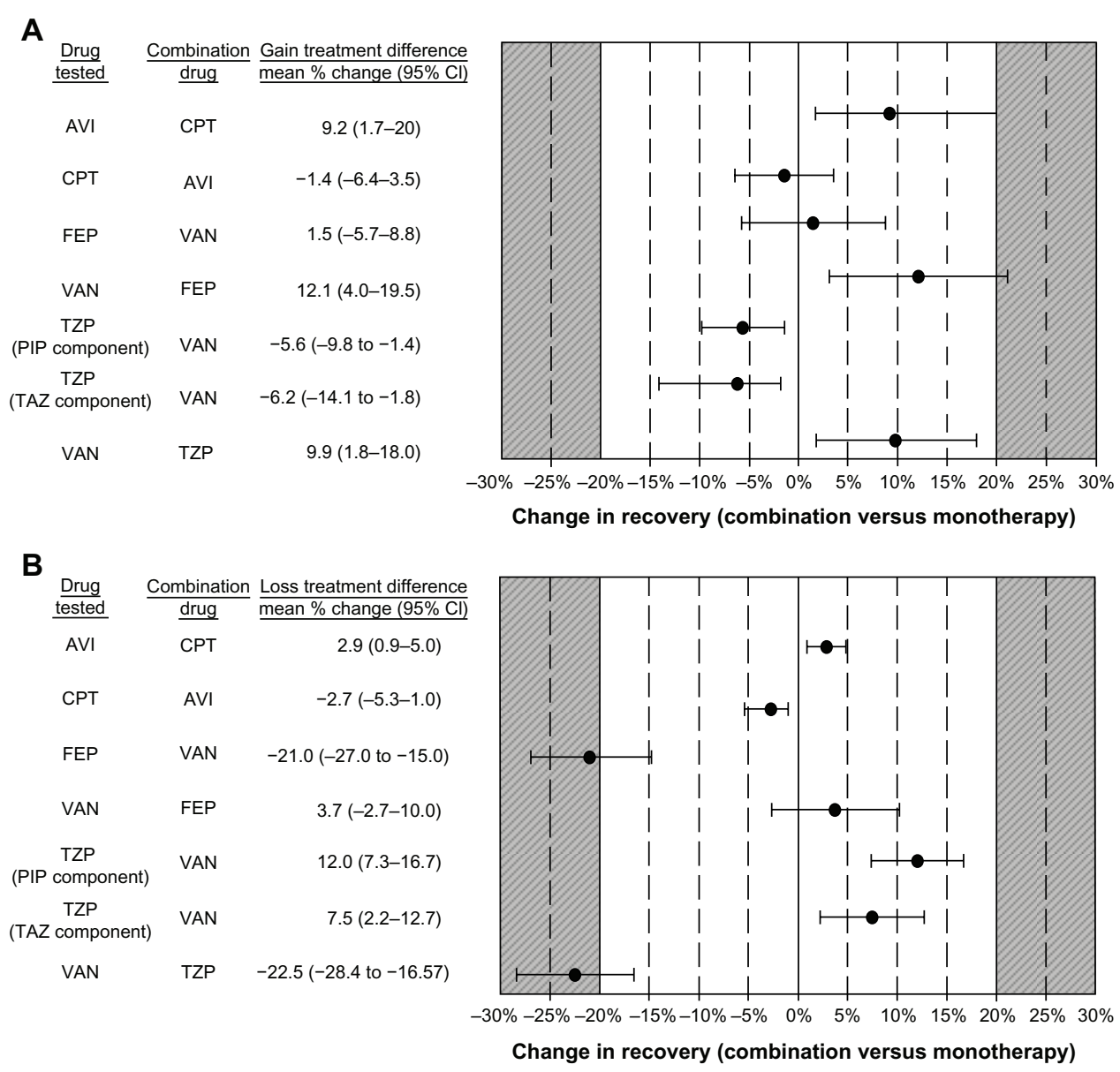

Figure I Change in mean recovery by gain (A) and loss (B) of compounds when administered alone and in combination with another antibiotic. Notes: Circles represent mean difference in recovery between combination and monotherapy. Bars represent $95 \%$ confidence intervals.

Abbreviations: AVI, avibactam; CPT, ceftaroline fosamil; FEP, cefepime; PIP, piperacillin; TAZ, tazobactam; TZP, piperacillin-tazobactam; VAN, vancomycin; Cl, confidence interval.

vancomycin has the largest molecular weight, ${ }^{8}$ this may have contributed to the observed reduction in recovery by loss when administered in the presence of another antibiotic. Taking this into consideration, it is reasonable that the presence of multiple compounds during retrodialysis (recovery by loss) may have an impact on the recovery of others in the perfusion fluid. For instance, the reduced recovery by loss observed for cefepime (when combined with vancomycin) and vancomycin (when combined with piperacillin-tazobactam) in these studies would have led to an overestimation of their relative recovery.

We used a perfusion rate of $2 \mu \mathrm{L} / \mathrm{min}$ for the current in vitro experimentation to mimic the conditions under which the retrodialysis procedure is performed during in vivo microdialysis studies. Importantly, there was no observed difference, based on our a priori definition, between single and two-drug combinations for recovery by gain. The explanation for the alteration in recovery by gain but not for loss may be attributed to the relative difference in drug-containing solutions used in the experiments. The small volume of the drug-containing solutions required for the retrodialysis experiments $(\sim 120 \mu \mathrm{L} / \mathrm{h})$ is roughly 100 -fold smaller compared with the relatively large volume for gain $(10 \mathrm{~mL})$.

Although not directly translatable to in vivo studies, a similar phenomenon is observed when comparing the volume of retrodialysis solution with the volume of distribution seen in patients during the microdialysis procedure. This suggests that at concentrations higher than routinely observed in humans, the presence of two antibiotics in vivo is not anticipated to influence the recovery of one or the other, based on these in vitro findings.

\section{Conclusion}

In conclusion, we propose in vitro studies of two drugs in combination should be conducted prior to performing in vivo retrodialysis in patients, to confirm similar recovery. In doing so, we considered $\leq 20 \%$ difference between single and twodrug combinations to be an acceptable level of variance for the studied drug. 


\section{Acknowledgments}

We thank Christina Sutherland for her assistance with HPLC analysis. Additionally, we thank Seema Garg, from Eurofins Scientific Inc., for her work on the ceftaroline fosamil and avibactam assay. This work was supported internally by the Center for Anti-Infective Research and Development.

\section{Disclosure}

DPN has received grant support from Forest Laboratories, Inc. SHM and STH report no conflicts of interest relevant to this article.

\section{References}

1. Melgaard L, Hersini KJ, Gazerani P, Petersen LJ. Retrodialysis: a review of experimental and clinical applications of reverse microdialysis in the skin. Skin Pharmacol Physiol. 2013;26:160-174.

2. de Lange EC, de Boer AG, Breimer DD. Methodological issues in microdialysis sampling for pharmacokinetic studies. Adv Drug Deliv Rev. 2000;45:125-148.

3. Elmquist WF, Sawchuk RJ. Application of microdialysis in pharmacokinetic studies. Pharm Res. 1997;14:267-288.

4. Ståhle L, Arner P, Ungerstedt U. Drug distribution studies with microdialysis. III: Extracellular concentration of caffeine in adipose tissue in man. Life Sci. 1991;49:1853-1858.

5. Chaurasia CS, Müller M, Bashaw ED, et al. AAPS-FDA workshop white paper: microdialysis principles, application, and regulatory perspectives. J Clin Pharmacol. 2007;47:589-603.
6. Barbhaiya RH, Forgue ST, Shyu WC, Papp EA, Pittman KA High-pressure liquid chromatographic analysis of BMY-28142 in plasma and urine. Antimicrob Agents Chemother. 1987;31:55-59.

7. Hagihara M, Sutherland C, Nicolau DP. Development of HPLC methods for the determination of vancomycin in human plasma, mouse serum and bronchoalveolar lavage fluid. J Chromatogr Sci. 2013;51:201-207.

8. Kim MK, Capitano B, Mattoes HM, et al. Pharmacokinetic and pharmacodynamic evaluation of two dosing regimens for piperacillin-tazobactam. Pharmacotherapy. 2002;22:569-577.

9. Wiskirchen DE, Crandon JL, Furtado GH, Williams G, Nicolau DP. In vivo efficacy of a human-simulated regimen of ceftaroline combined with NXL104 against extended-spectrum-beta-lactamase (ESBL)producing and non-ESBL-producing Enterobacteriaceae. Antimicrob Agents Chemother. 2011;55:3220-3225.

10. Joukhadar C, Müller M. Microdialysis: current applications in clinical pharmacokinetic studies and its potential role in the future. Clin Pharmacokinet. 2005;44:895-913.

11. Infectious Diseases Society of America. Guidelines for the management of adults with hospital-acquired, ventilator-associated, and healthcare-associated pneumonia. Am J Respir Crit. Care Med. 2005;171:388-416.

12. Klibanov OM, Raasch RH, Rublein JC. Single versus combined antibiotic therapy for gram-negative infections. Ann Pharmacother. 2004;38: 332-337.

13. Infectious Diseases Society of America. The $10 \mathrm{x}$ '20 Initiative: pursuing a global commitment to develop 10 new antibacterial drugs by 2020 . Clin Infect Dis. 2010;50:1081-1083.

14. Boucher HW, Talbot GH, Benjamin DK Jr, et al. $10 \times$ '20 Progressdevelopment of new drugs active against gram-negative bacilli: an update from the Infectious Diseases Society of America. Clin Infect Dis. 2013;56:1685-1694.
Clinical Pharmacology: Advances and Applications

\section{Publish your work in this journal}

Clinical Pharmacology: Advances and Applications is an international, peer-reviewed, open access journal publishing original research, reports, reviews and commentaries on all areas of drug experience in humans. The manuscript management system is completely online and includes a very quick and fair peer-review system, which is all easy to use

\section{Dovepress}

Visit http://www.dovepress.com/testimonials.php to read real quotes from published authors. 